\title{
Highly flexible, stretchable, patternable, transparent copper fiber heater on a complex 3D surface
}

\author{
Hong Seok Jo ${ }^{1,4}$, Seongpil An ${ }^{1,4}$, Jong-Gun Lee ${ }^{1}$, Hyun Goo Park ${ }^{1}$, Salem S Al-Deyab ${ }^{2}$, Alexander L Yarin ${ }^{1,3}$ \\ and Sam S Yoon ${ }^{1}$
}

\begin{abstract}
A highly transparent, flexible, stretchable and patternable copper fiber heater was successfully fabricated for potential use in smart windows and other applications. The thickness of the electrospun polymer nanofibers was controlled during subsequent copper plating by adjusting the electroplating time, with minimal sacrifice in transparency. Self-fused junctions, formed via electroplating, significantly reduced the contact resistance between the intersecting copper-plated fibers. The heater temperature remained constant up to $300 \%$ sheet stretching. De-icing tests confirmed the potential applicability of such heaters in smart windows or vehicle defrosters. The copper-plated fibers may be transferred onto any surface with a complex 3D structure, as demonstrated by fabricating a heat-radiating Venus statue covered with the copper-plated fibers. The highest temperature of $328{ }^{\circ} \mathrm{C}$ was achieved by using a transparent fibrous film having $90 \%$ transparency and $0.058 \Omega \mathrm{sq}^{-1}$ sheet resistance. NPG Asia Materials (2017) 9, e347; doi:10.1038/am.2016.206; published online 10 February 2017
\end{abstract}

\section{INTRODUCTION}

Transparent heaters are important for various applications under cold and icy conditions, such as solar panels, vehicle defrosters, and smart, heat-retaining windows. ${ }^{1-11}$ They are also used in home appliances such as transparent toasters, water heaters, ${ }^{12}$ and portable home air heaters. To meet the requirements of high transparency and high electrical conductivity, nanomaterials including graphene, ${ }^{13,14}$ carbon nanotubes (CNTs), ${ }^{15,16}$ and conducting polymers ${ }^{17}$ have been used in such heaters. ${ }^{18}$ Electrospun fibers were coated with copper by high temperature thermal evaporation and sputtering for heater applications. While these nanomaterials are ideal for obtaining high transparency and offer the benefit of low material consumption rates, they are often expensive and require multiple fabrication steps, which increase manufacturing costs. Above all, the most critical issue is the limited power $(P)$ that can be supplied to such materials. This severely restricts the maximum attainable temperature $\left(T_{\mathrm{s}}\right)$, since $T_{\mathrm{s}} \propto P$. For example, the temperature ranges of graphene heaters, silver nanowire heaters, and heaters utilizing hybrid materials combining graphene and silver nanowires are $100-206^{\circ} \mathrm{C},{ }^{19-21} 48-160^{\circ} \mathrm{C},{ }^{22-27}$ and up to $225^{\circ} \mathrm{C},{ }^{28,29}$ respectively.

Recently, An et al. ${ }^{30}$ achieved record transparency and sheet resistance using electrospun copper-plated fibers. The size of the fibers was in the $1-2 \mu \mathrm{m}$ range, which facilitated rapid and efficient electron transport. Furthermore, the contact resistance between these fibers was significantly reduced by fusing the fibers into a single unit via copper electroplating. The use of copper as a heating material offers two major advantages. First, the costs are low compared with those of carbon nanotubes (CNTs), graphene, or silver nanowires. Secondly, copper can yield higher heating temperatures because it has a higher melting temperature than that of silver nanowires, as well as a higher electrical conductivity because of the microscale thickness of the copper fibers, which allows the transport of more electrons than occurs in nanoscale wires. The copper fibers developed by An et al. ${ }^{30}$ were also freestanding, and thus can be readily transported onto surfaces with complex shapes.

Herein, nano- and microscale copper-plated fibers are utilized to achieve heating temperatures exceeding $300^{\circ} \mathrm{C}$. The developed heating film is transparent, flexible, stretchable, and can be deposited onto any complex three-dimensional surface.

\section{EXPERIMENTAL PROCEDURES}

Materials

The electrospinning solution for fabricating polymer nanofibers was prepared by dissolving $8 \mathrm{wt} \%$ polyacrylonitrile (PAN, $M_{\mathrm{w}}=150 \mathrm{kDa}$, Sigma-Aldrich) using N,N-dimethylformamide (DMF, 99.8\%, Sigma-Aldrich, St Louis, MO, USA) as a solvent. The solution was then stirred for several hours at room temperature until the PAN was fully dissolved. The electroplating solution for copper-plating the polymer nanofibers was prepared by mixing $25 \mathrm{~g}$ of sulfuric acid (Matsunoen Chemicals, Osaka, Japan), $2.5 \mathrm{~g}$ of hydrochloric acid (SigmaAldrich), $80 \mathrm{~g}$ of copper sulfate (Sigma-Aldrich), $50 \mathrm{~g}$ of formaldehyde (SigmaAldrich), and $500 \mathrm{ml}$ of deionized (DI) water and stirring for $1 \mathrm{~h}$ at room temperature.

${ }^{1}$ School of Mechanical Engineering, Korea University, Seoul, Republic of Korea; ${ }^{2}$ Petrochemicals Research Chair, Department of Chemistry, King Saud University, Riyadh, Saudi Arabia and ${ }^{3}$ Department of Mechanical and Industrial Engineering, University of Illinois at Chicago, Chicago, IL, USA

${ }^{4}$ These authors contributed equally to this work.

Correspondence: Professor A Yarin, Department of Mechanical and Industrial Engineering, University of Illinois at Chicago, 842 W. Taylor St, Chicago, IL 60607, USA.

E-mail: ayarin@uic.edu

or Professor S Yoon, School of Mechanical Engineering, Korea University, 145 Anam-ro, Seongbuk-gu, Seoul 02841, Republic of Korea.

E-mail: skyoon@korea.ac.kr

Received 12 July 2016; revised 11 November 2016; accepted 16 November 2016 

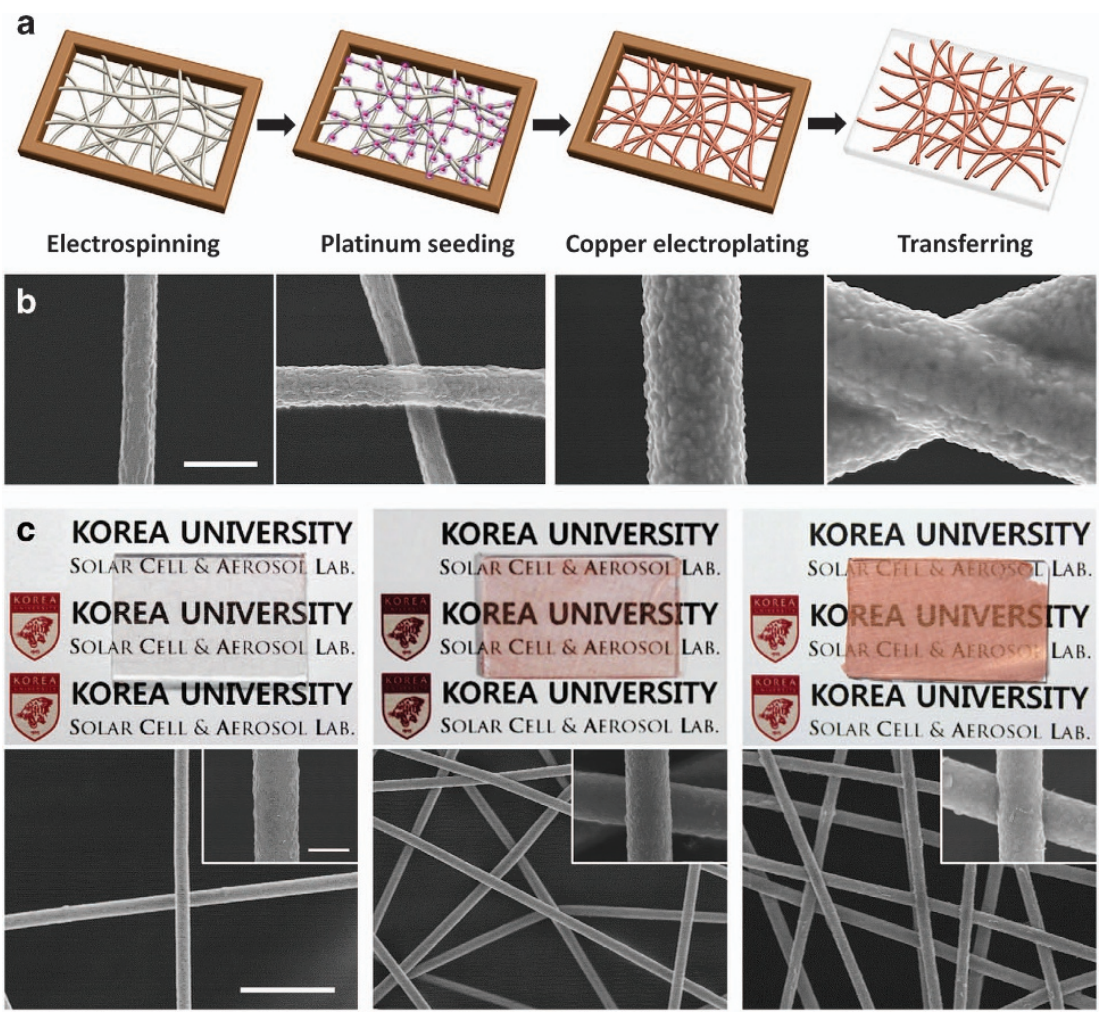

Figure 1 (a) schematic of the CFH fabrication process. (b) SEM images of pristine PAN, platinum-seeded, Cu-electroplated, and transferred nanofibers. The scale bar is $1 \mu \mathrm{m}$. (c) Photographs and corresponding SEM images of the CFHs on glass substrates with first electrospinning times of 5, 90, and 180 s. The scale bars are $10 \mu \mathrm{m}$; those for the insets are $1 \mu \mathrm{m}$.

\section{Fabrication of copper nanofibers}

In brief, PAN nanofibers were first electrospun onto a square copper frame for the electrospinning times $\left(t_{\mathrm{s}}\right)$ of 5,90 , or $180 \mathrm{~s}$ at a rate of $150 \mu \mathrm{h} \mathrm{h}^{-1}$ and a voltage of $5 \mathrm{kV}$. For electrospinning, a needle (25 gauge, EFD), syringe pump (Legato 100, KD Scientific, Holliston, MA, USA), and DC power supply (EL20P2, Glassman high voltage) were used; see Figure 1a. Then, platinum (Pt) seeds were sputtered onto the nanofibers to make them conductive and facilitate the subsequent copper-plating process. After that, the second layer of electrospun polymer nanofibers was deposited above the Pt-seeded nanofibers. Only the Pt-seeded nanofibers are electroplated by copper. The second layer of electrospun nanofibers was not electroplated because without Pt seeding these nanofibers are non-conductive. Further on, the square frame with nanofibers (the cathode) was copper-plated using a pure copper frame (the anode) at an applied voltage of $3 \mathrm{~V}$. Because $t_{\mathrm{s}}$ was varied, different plating times $\left(t_{\mathrm{p}}\right)$ were used to achieve uniform copper fiber (CF) diameters. At the following step, the square frame was immersed in $10 \%$ formaldehyde for $5 \mathrm{~min}$ and then rinsed with DI water. After the rinsing, the copper fibers were transferred onto glass and then the non-seeded second-layer polymer nanofibers were removed by DMF dissolution. Finally, the copper fibers were dried in a nitrogen-filled chamber for a few minutes. More details on some of the above-mentioned operations were reported in our previous studies. ${ }^{31,32}$

\section{Characterization}

The morphologies of PAN and the CuNFs were analyzed using scanning electron microscopy (SEM, S-5000, Hitachi, Tokyo, Japan). The transmittance and the sheet resistance of the copper fiber film heater (CFH) were measured using a UV-Vis spectrophotometer (Optizen POP, Mecasys, Daejeon, Korea) and a sheet resistance meter (FPP-400, Dasol Eng, Cheongju, Korea) as shown in Supplementary Movie S1, respectively. The surface temperature of the CFH was measured by a thermocouple with a data recorder (MV1000, Yokogawa, Tokyo, Japan) and an IR camera (FLIR-E63900, FLIR, Wilsonvile, OR, USA).
Whether the CFHs were oxidized or not was identified using energy dispersive X-ray spectrometry (EDX, S-4300, Hitachi). Bending and stretching tests were conducted using an in-house device.

\section{RESULTS AND DISCUSSION \\ Copper fibers}

The overall fabrication process for the $\mathrm{CFH}$ is illustrated in Figure 1a. PAN is electrospun to form networks of suspended nanofibers on the square copper frame, which creates an inter-connected nanofiber network with many inter-fiber openings (two left SEM images in Figure 1b). After copper-plating, this unique network of nanofibers possesses a high electrical conductivity and light transmittance, appropriate for use as a transparent heater $(\mathrm{CFH})$. Prior to the copperplating, platinum seeding was conducted using a sputter, as described in detail in ref. 30 As shown in the two right SEM images in Figure 1b, copper-plating creates self-fused fiber junctions at the intersections of the original polymer nanofibers, which significantly reduce the sheet resistance. The overall results obtained with the fabricated $\mathrm{CFH}$ are elucidated in the following sections. Additionally, once the CFH was dried under nitrogen atmosphere, oxidation was avoided without deteriorating the performance, even under continuous exposure to air.

\section{Optical and electrical properties}

Figure $2 \mathrm{c}$ show macroscopic and SEM images of the CFHs on heat resistant glass $\left(3 \times 2 \times 0.7 \mathrm{~mm}^{3}\right)$ with varied first electrospinning times $\left(t_{\mathrm{s}}\right)$. Increasing $t_{\mathrm{s}}$ from 5 to $180 \mathrm{~s}$ reduces the transmittance $(T)$ of the CFHs from 96 to $30 \%$ because of the light-scattering effect, which becomes dominant as the number of copper fibers increases (Supplementary Figure S1). In contrast, the sheet resistance $\left(R_{\mathrm{S}}\right)$ is 
a

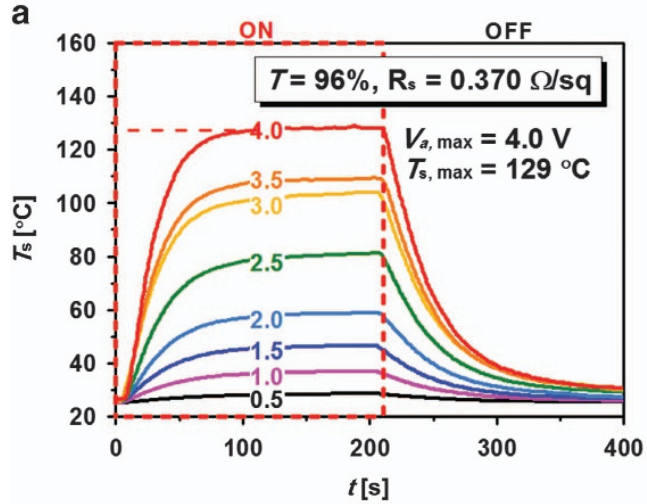

C

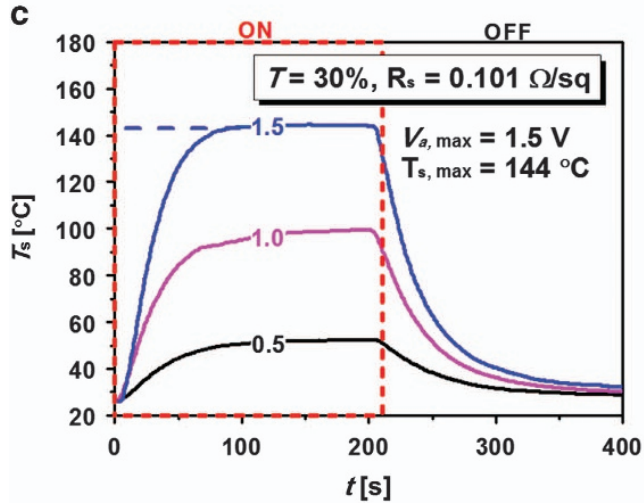

b

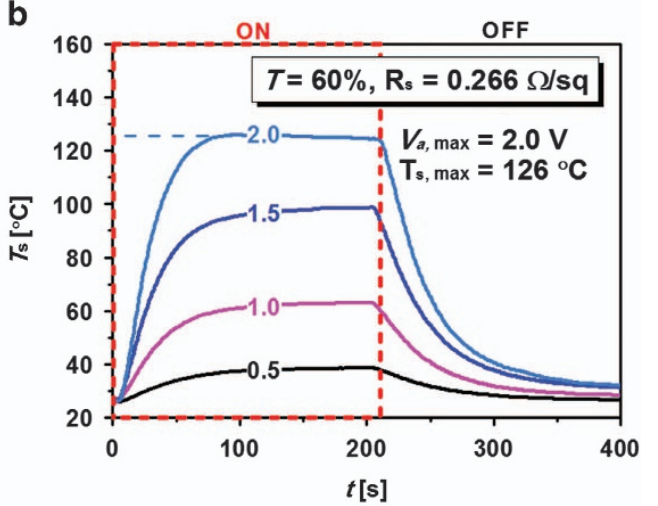

d

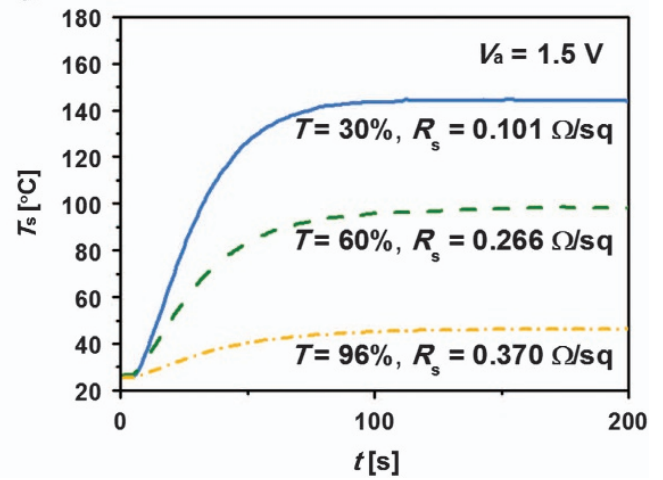

Figure 2 Temperature profiles of the CFH with (a) $T=96 \%$, (b) $T=60 \%$, and (c) $T=30 \%$ at different applied voltages. (d) Temperature profiles reaching steady state for different $T$ values at a fixed $V_{a}$ of $1.5 \mathrm{~V}$. In a-c, the numbers on the curves denote the corresponding values of the applied voltage $V_{a}$. In a-c, heating is turned off at $t=200 \mathrm{~s}$.

decreased from 0.370 to $0.101 \Omega \mathrm{sq}^{-1}$ with increases in $t_{\mathrm{s}}$ because of the reduced charge separation distance (Figure 1c, Supplementary Table S1).

Different electroplating times $\left(t_{\mathrm{p}}\right)$ are used in the present work, in contrast to our previous work. ${ }^{30}$ In the previous study, the electroplated copper mass increased by $\sim 1.9$-fold when $t_{\mathrm{s}}$ was increased from 1 to $180 \mathrm{~s}$ at a fixed $t_{\mathrm{p}}$. To produce copper fibers with uniform diameters in all cases in this study, an optimized $t_{\mathrm{p}}$ was used (Supplementary Table S1). The optimized $t_{\mathrm{p}}$ was established using the electric charge conservation law. The electric charge per unit area should be constant over the entire surface to obtain uniform fiber diameters, regardless of $t_{\mathrm{s}}$. For this reason, the charge in Coulombs per micrometer square $\left(C_{a}\right)$ was considered, and its value was calculated for various $t_{\mathrm{p}}$ values. It was found that $t_{\mathrm{p}}=2,5$, and $10 \mathrm{~s}$ were adequate for $t_{\mathrm{s}}=5,90$, and $180 \mathrm{~s}$, respectively, as noted in Supplementary Table S1, and the corresponding $C_{a}$ values were found as below, following the conservation of electric charge. ${ }^{33}$

$$
C_{\mathrm{a}}=\frac{I_{\mathrm{p}} t_{\mathrm{p}}}{\pi D_{\mathrm{f}} L}
$$

Here, $I_{\mathrm{p}}$ is the electroplating current and $t_{\mathrm{p}}$ is the electroplating time. $D_{\mathrm{f}}$ and $L$ are the average diameter of the PAN nanofibers and the total length of the PAN nanofibers in the projected area of $20 \times 20 \mu \mathrm{m}^{2}$, respectively.

As listed in Supplementary Table S2, even though $L$ and $t_{\mathrm{s}}$ change dramatically for the different cases, the $C_{\mathrm{a}}$ values remain constant because of the optimized $t_{\mathrm{p}}$. Thus, the average diameter of the copper fibers $\left(D_{\mathrm{p}}\right)$ for the different cases is almost constant, within the $1.16-1.19 \mu \mathrm{m}$ range.

\section{Heating performance}

To compare the heating performance of the CFHs as a function of the transmittance $T$ and the applied voltage $\left(V_{\mathrm{a}}\right)$, the time-dependent temperature profiles of the $\mathrm{CFHs}$ were measured as depicted in Figure 2. The size of all tested CFH films was fixed to $2 \times 3.2 \mathrm{~cm}^{2}$. While varying $V_{\mathrm{a}}$ and hence the power $P$, a thermocouple was embedded at the upper surface of the $\mathrm{CFH}$ coated on the heat resistant glass to measure the temperature $T_{\mathrm{s}}$.

The steady-state temperature $\left(T_{\mathrm{s}, \max }\right)$ in all cases shown in Figures $2 \mathrm{a}-\mathrm{c}$ increases as the applied voltage $V_{\mathrm{a}}$ increases, because the power $P$ is supplied to the heaters as Joule heat, which means that $P=V_{\mathrm{a}}^{2} / R$, with $R$ being the total resistance. At higher transmittance values $T$, the heater surface has fewer copper-plated fibers, and thus a higher voltage $V_{\mathrm{a}}$ should be applied to achieve a surface temperature $T_{\mathrm{s}}$ similar to that at lower- $T$ values. For example, $T_{\mathrm{s}}$ reaches $\sim 130{ }^{\circ} \mathrm{C}$ when $V_{\mathrm{a}}$ is increased to $4 \mathrm{~V}$ for $T=96 \%$, whereas a close $T_{\mathrm{s}}$ value of $126^{\circ} \mathrm{C}$ is achieved at only $V_{\mathrm{a}}=2 \mathrm{~V}$ for $T=60 \%$. Similarly, $T_{\mathrm{s}}$ increases above $140{ }^{\circ} \mathrm{C}$ at $V_{\mathrm{a}}=1.5 \mathrm{~V}$ for $T=30 \%$. In addition, $T_{\mathrm{s}}$ increases as $R$ is lower at the equal voltage in Figure $2 \mathrm{~d}$.

The overall Joule heat $(H)$ released by a heater during time $\Delta t$ is $H=I V \Delta t$, where $I=V_{\mathrm{a}} / R$ is the electric current. At a given applied voltage $V_{\mathrm{a}}$, the resistance $R$ should be low to achieve a high electric current. Thus, the extremely low resistance of the CFHs, with a sheet resistance of $0.101 \leqslant R_{\mathrm{s}} \leqslant 0.370 \Omega \mathrm{sq}^{-1}$, greatly contributes to the outstanding heating performance compared with that achieved in 
Table 1 Properties of transparent film heaters using different materials

\begin{tabular}{lccccc}
\hline Nanomaterials & $V_{a, \max }(V)$ & $R_{s}\left(\Omega s q^{-1}\right)$ & $T(\%)$ & $\left.T_{s, \max }{ }^{\circ} \mathrm{C}\right)$ & Reference \\
\hline MWCNT & 15 & 699 & 83 & 77 & 34 \\
MWCNT & 40 & 756 & 90 & 102 & 35 \\
SWCNT & 60 & 136 & 70 & 160 & 36 \\
SWCNT & 60 & 580 & 79 & 120 & 37 \\
Graphene-AuCl & 12 & 43 & 89 & 100 & 21 \\
rGO & 60 & 641 & 34 & 206 & 20 \\
Graphene-AuCl & 12 & 66 & 90 & 110 & 19 \\
Ag mesh & 12 & 300 & 88 & 43 & 38 \\
AgNW-ITO & 5 & 4.1 & 83 & 70 & 39 \\
CuNi mesh & 9 & 16.2 & 86 & 225 & 40 \\
Cu mesh & 0.21 & 0.3 & 78 & 80 & 41 \\
Cu-plated fibers & 1.5 & 0.058 & 90 & 328 & Present \\
\hline
\end{tabular}

Abbreviations: MWCNT, multi-walled carbon nanotubes; SWCNT, single-walled carbon nanotubes.

prior studies with single-walled CNTs, graphene, reduced graphene oxide, silver nanowires, and copper mesh (Table 1).

Note also that, according to Joule's law, the released electric power is

$$
P=V_{\mathrm{a}} I \text {. }
$$

This power is spent for heating the film heater and the convective, conductive, and radiative heat losses from the film, which means that

$$
V_{\mathrm{a}} I=c m \frac{\mathrm{d} T_{\mathrm{s}}(t)}{\mathrm{d} t}+Q_{\text {loss }}
$$

where $c$ and $m$ are the specific heat and mass of the heater, and the term $Q_{\text {loss }}$ describes the overall thermal losses. In the present case, the radiative heat loss is negligible compared with the conductive heat loss, as the surface temperatures are below $300^{\circ} \mathrm{C}$. The conductive losses to the glass substrate and the surrounding are also negligibly small. Therefore, $Q_{\text {loss }}$ can be expressed as

$$
Q_{\text {loss }}=h A\left(T_{\mathrm{s}}-T_{\mathrm{a}}\right)
$$

where $h$ is the convective heat transfer coefficient, $A$ is the projected surface area of the film heater, and $T_{\mathrm{a}}$ is the ambient air temperature. Thus, the solution of Equations (3) and (4) yields

$$
T_{s}=\frac{V_{a} I}{h A}\left(1-e^{-\frac{h A}{c m} t}\right)+T_{a}+\left(T_{\mathrm{i}}-T_{\mathrm{a}}\right) e^{-\frac{h A}{c m} t}
$$

where $T_{\mathrm{i}}$ is the initial temperature at $t=0$ when heating begins.

The corresponding steady-state temperature is found from Equation (5) as

$$
T_{s, \max }=\frac{V_{\mathrm{a}} I}{h A}+T_{\mathrm{a}} .
$$

By using Equation (6), the values of $h$ could be obtained as $h=V_{\mathrm{a}} I /$ $\left[\left(T_{\mathrm{s}, \max }-T_{\mathrm{a}}\right) A\right]$. Then, using the results plotted in Figures $2 \mathrm{a}-\mathrm{c}$, the values of $h$ found at $T=30,60$, and $96 \%$ are similar, as in Figure 3a, but the lowest $T_{\mathrm{s}, \max }$ value corresponds to the largest $h$ value. The comparison between the experimental and theoretical dependences of the surface temperature on the power supply presented in Figure 3a reveals an excellent agreement.

In Figure $3 \mathrm{~b}$ and Supplementary Figure S2, the thermal stability and durability of the CFHs with different values of transmittance $T$ were evaluated for 20 cycles of the applied voltage to achieve temperature variation in the $30{ }^{\circ} \mathrm{C} \leqslant T_{s} \leqslant 107^{\circ} \mathrm{C}$ range (Figure $3 \mathrm{~b}$ and Supplementary Figure S2). The heating performance remains constant even after 20 cycles. It is noteworthy that copper oxidation appeared to be negligible, as shown in Supplementary Table S3. Figure 3c compares the temperature rise of the CFHs of various transmittance values $(T)$. The film with higher transparency requires a longer time and greater voltage to reach the same $T_{\mathrm{s}}=185^{\circ} \mathrm{C}$. The samples with higher transparency correspond to more open heater structures, and thus higher resistances $R$ or lower electric currents $I$ for the same voltage. Therefore, to release the same power in Joule heating, and thus reach the same temperature $T_{s, \max }$, the more transparent samples require higher applied voltages, since $P=V_{\mathrm{a}}^{2} / R=I V_{\mathrm{a}}$.

The optical and electrical performance can be significantly improved by changing $t_{\mathrm{s}}$ and $t_{\mathrm{p}}$ slightly. The cross-sectional diameter of the copper fibers and their fusion at the intersections could be increased; both changes facilitate reduction in the electrical resistance. Figure $3 \mathrm{~d}$ and Supplementary Table S4 show the enhanced heating performance in the four cases with variation of $t_{\mathrm{s}}$ and $t_{\mathrm{p}}$. The surface temperature $T_{\mathrm{s}}$ increased significantly from 185 to $284^{\circ} \mathrm{C}$ as $t_{\mathrm{p}}$ was increased from 2 to $12 \mathrm{~s}$ when $t_{\mathrm{s}}$ was fixed at $5 \mathrm{~s}$ (Supplementary Table S4). The corresponding transmittance $T$ and resistance $R_{\mathrm{S}}$ were decreased from 96 to $83 \%$ and from 0.370 to $0.076 \Omega \mathrm{sq}^{-1}$, respectively, because of the increased diameters of the fibers from Case 1 to Case 4 , showing a change in $D_{\mathrm{f}}$ from 1.7 to $9 \mu \mathrm{m}(430 \%$ increase). It should be emphasized that the surface temperature $T_{\mathrm{s}}$ of the CFH could exceed $300^{\circ} \mathrm{C}$ even at $t_{\mathrm{s}}=3 \mathrm{~s}$ when $t_{\mathrm{p}}$ was increased to 15 s. Notably, from Supplementary Table S4, an increase of $\sim 5.3$ times in the fiber diameter resulted in a decrease of $\sim 5.3$ times $(0.308 / 0.058)$ in the sheet resistance. Having thick microfibers decreases the sheet resistance significantly, and yet the transmittance is not significantly sacrificed as long as the electrospinning time is moderately reduced, providing enough pores for efficient light transmittance.

\section{Flexibility and stretchability}

We have so far tested the CFH deposited on rigid non-flexible glass substrates. To demonstrate the flexibility and stretchability of the $\mathrm{CFH}$, the fibers were deposited on flexible and stretchable substrates of 0.01mm-thick PET and stretchable 2-mm-thick Ecoflex rubber. Ecoflex rubbers are transparent, extremely stretchable silicone-based materials. Using these substrates, the mechanical properties of the CFHs can be varied, which is the most important factor for the application of the film heaters in wearable devices. To evaluate the mechanical performance of the CFHs, bending and stretching tests were performed using the CFH with $T=96 \%$ on flexible PET and stretchable Ecoflex substrates. The cyclic bending test was conducted with a bending radius of $R_{\mathrm{b}}=1.3 \mathrm{~mm}$ for 1000 cycles at a fixed applied voltage $V_{\mathrm{a}}$ of $2.5 \mathrm{~V}$ with the heat-radiating film, as shown in Figure 4a. The nominal bending strain, $\varepsilon$, is defined as $\varepsilon=d / R_{\mathrm{b}}$, where $d$ is the substrate thickness. ${ }^{22}$ In the present case, $\varepsilon$ is 0.01 for the CFH with $T=96 \%$. The corresponding resistance change is expressed as $\Delta R_{s}=\left(R_{\mathrm{s}}-R_{\mathrm{s} 0}\right) /$ $R_{\mathrm{s} 0}$, where $R_{\mathrm{s} 0}$ is the initial resistance and $R_{\mathrm{s}}$ is the resistance after the bending test. Supplementary Figure $\mathrm{S} 3$ reveals $\Delta R_{\mathrm{s}}=0.035$. This exceptional flexibility of the $\mathrm{CFH}$, as shown by its resistance retention after bending, is advantageous for various industrial applications.

Figure $4 \mathrm{~b}$ illustrates the stretchability of the $\mathrm{CFH}$ under uniaxial strain and a constant applied voltage. The change in the resistance and temperature at $V_{\mathrm{a}}=2.5 \mathrm{~V}$ are recorded as the film is stretched from 0 to $300 \%$. The $\mathrm{CFH}$ rapidly responds to the applied voltage, quickly reaching a steady-state temperature. Although the sample is stretched in one direction, the hot spot remains heated during the stretching of the Ecoflex substrate. Supplementary Figures S3 and 4 demonstrate the repeatability in the performance of the CFH film. The bending test up to 1000 cycles and the corresponding constant surface temperature 

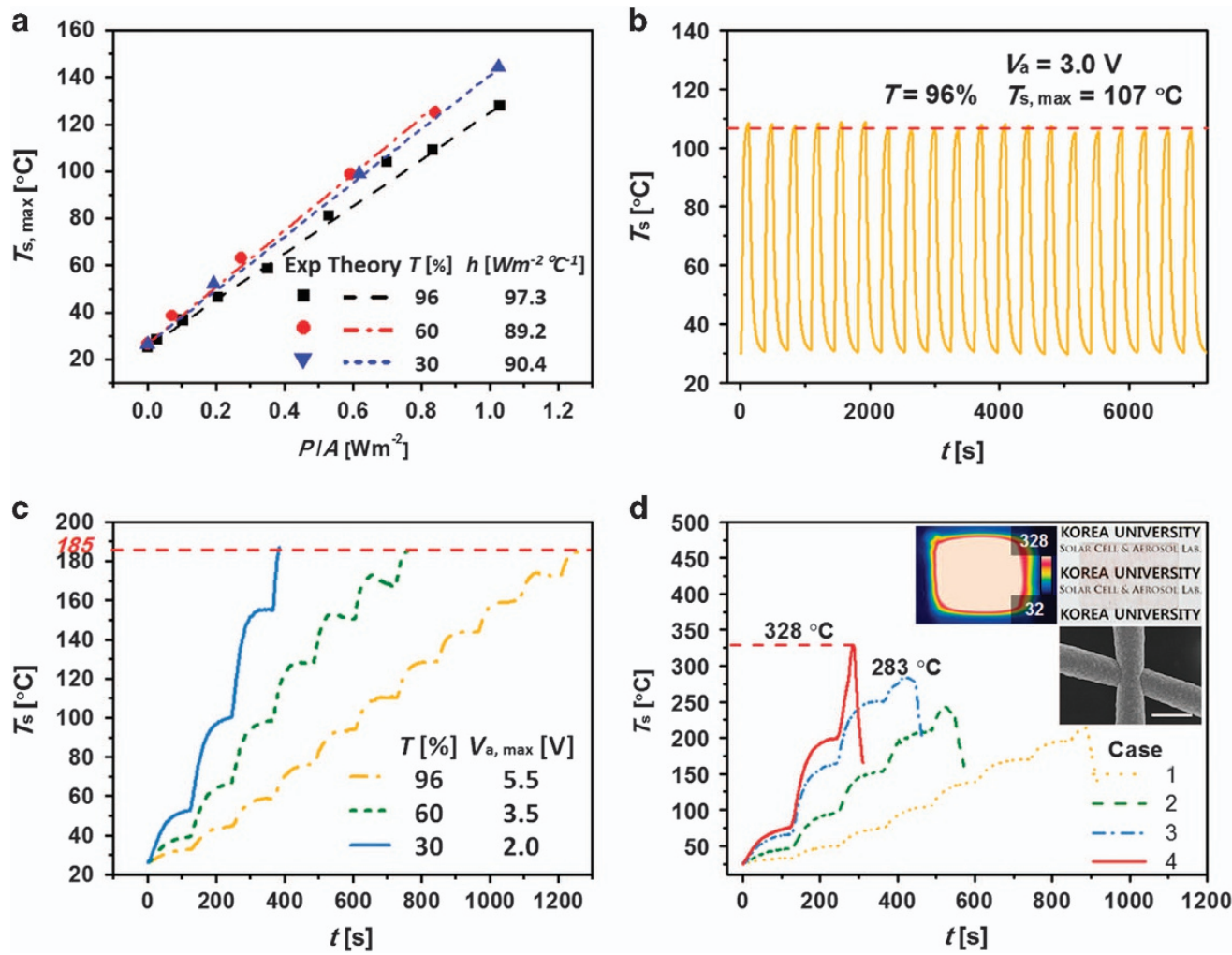

Figure 3 (a) The values of the heat-transfer coefficient $h$ evaluated for heaters with different transmittances $T$ and the comparison of the experimental and theoretical results for $T_{\mathrm{s}, \max }$ versus P/A for all cases. (b) Thermal stability of the CFHs with $T=96 \%$, evaluated by switching the applied voltage for 20 cycles. (c) Variation of temperature for CFHs with stepwise increases in the applied voltage. (d) Surface temperature $T_{\mathrm{s}}$ of the CFHs with stepwise increases in the applied voltage. Cases 1-4 are listed in Supplementary Table S4. The inset photograph and IR image correspond to case 4. The scale bar in the inset SEM image is $10 \mu \mathrm{m}$.
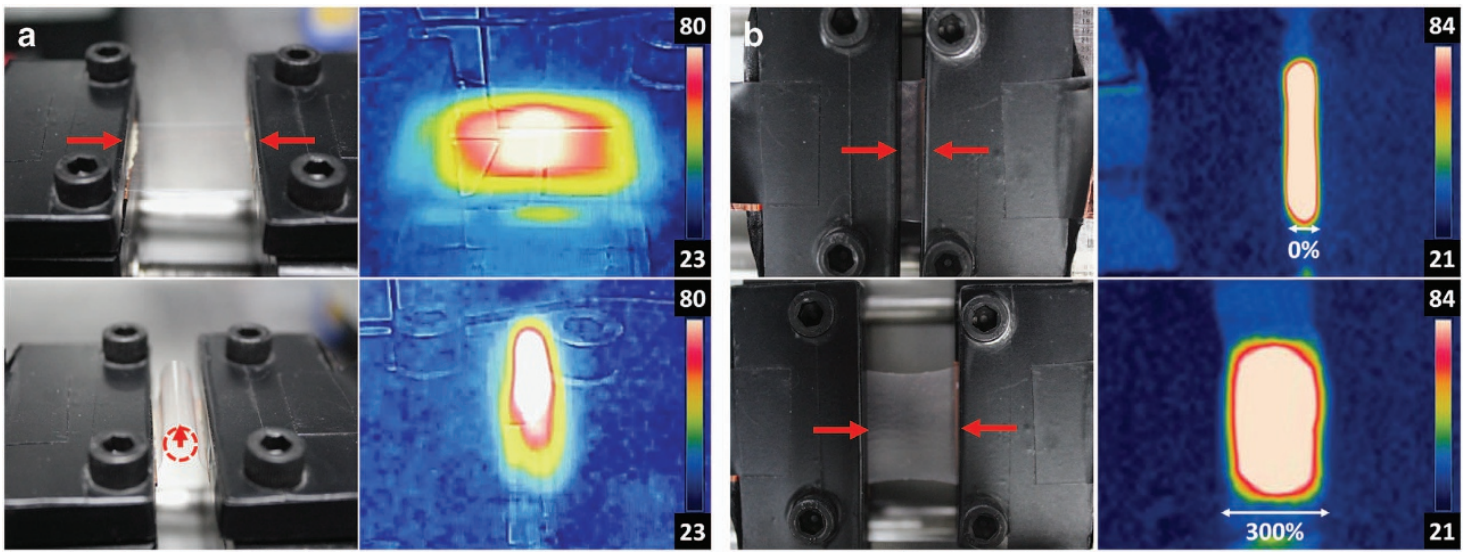

Figure 4 (a) Photographs and IR images of the CFH during the bending test at the initial state and the final state. The substrate used here is PET. (b) Photographs and IR images of the CFH during the stretching test at the initial state $(L=5 \mathrm{~mm})$ and the final state $(L=20 \mathrm{~mm})$. The transmittance of the CFH sample is $T=96 \%$. The substrate used here is Ecoflex rubber. The applied voltage is $V_{a}=2.5 \mathrm{~V}$ for both (a) and (b).

$\left(T_{\mathrm{s}}\right)$ in Supplementary Figure S3a confirm the mechanical sustainability of CFH. Likewise, the stretching test up to $300 \%$ also yields the robust mechanical stability of the heater, as shown in Supplementary Figure S3b. This stretching test was repeated up to 50 times and revealed only a minor change in $T_{s}$. Even after applying $300 \%$ stretching up to $N=50$ times, the film exhibits uniform heating. The corresponding SEM images also demonstrate that the copper fibers remain intact even after stretching. In particular, the fused intersections are intact, which sustain the high performance of the
CFH film. Supplementary Table S5 quantitatively compares the bendability and stretchability of various films. The present CFH films reveal only slight changes in temperature and sheet resistance after severe bending and stretching, demonstrating the superiority of our $\mathrm{CFH}$ films.

An additional method of evaluating the performance of a film heater is the defrosting test. Figure $5 \mathrm{a}$ reveals the defrosting capability of a CFH with the transmittance $T=96 \%$. A few water droplets were dripped onto the surface of the samples with and without $\mathrm{CFH}$. The 

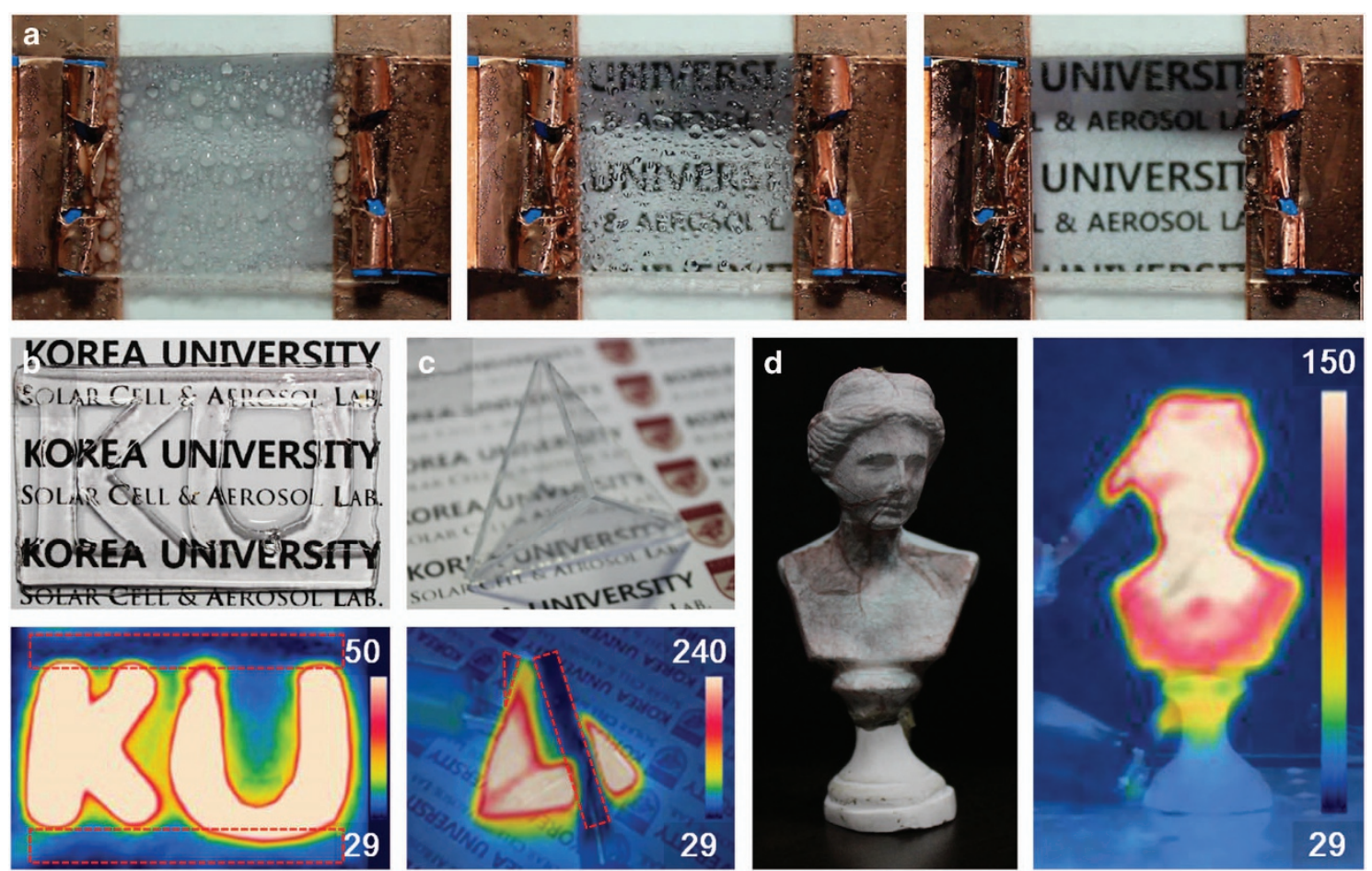

Figure 5 (a) Results of defrosting test with $\mathrm{CFH}$ with the transmittance $T=96 \%$ on a heat-resistant glass at $t=0,15$ and $40 \mathrm{~s}$ under $V_{\mathrm{a}}=3 \mathrm{~V}$. Photographs and IR images of (b) letter-patterned CFH at $V_{\mathrm{a}}=1.5 \mathrm{~V}$, (c) a pyramidal structure of a $3 \mathrm{D}$ heater at $V_{\mathrm{a}}=2.5 \mathrm{~V}$, and (d) a statue of Venus covered with copper fibers on the left, which undergoes Joule heating at $V_{a}=3.5 \mathrm{~V}$ as is shown by the IR image on the right.

wetted samples were then kept in a freezer for $30 \mathrm{~min}$ to form frost. When $V_{\mathrm{a}}=3 \mathrm{~V}$ was applied to the sample with $\mathrm{CFH}$, the frost on the surface of the CFH melted completely within $15 \mathrm{~s}$, and the resulting water was totally evaporated in $40 \mathrm{~s}$. Initially, the letters behind the frosted glass are invisible. However, after ice melting and water evaporation due to heat, the glass becomes transparent and the letters behind it become visible. The highly transparent $\mathrm{CFH}$ exhibits apparently high heating performance, demonstrating potential for applications such as outdoor advertisement boards, windshields, or vehicle defrosters.

The highly flexible and stretchable CFHs enable the fabrication of letter-patterned transparent heaters and complex three-dimensional surface heaters, as shown in Figures $5 \mathrm{~b}$ and c. A free-standing CFH with $T=96 \%$ was transferred onto a polydimethylsiloxane (PDMS) film. The film was cut and shaped to form the letters KU. A silver paste was deposited on both ends of the film. The red-dashed boxes on top and bottom of the letter ' $K$ ' and ' $U$ ' (shown in Figure 5b) are electrodes connected to the letters to supply voltages to them. The letter-patterned transparent heater rapidly responds to the electrical signal, achieving a surface temperature of $\sim 50^{\circ} \mathrm{C}$, which agrees with the results in Figure 2a. A CFH with $T=96 \%$ was also transferred onto a pyramidal structure (cf. Figure $5 \mathrm{c}$ ) with the voltage level of $V_{\mathrm{a}}=2.5 \mathrm{~V}$. The same $\mathrm{CFH}$ was also transferred onto a replica statue of Venus at $V_{\mathrm{a}}=3.5 \mathrm{~V}$. The fairly uniform temperature distributions were observed in both cases. The highest temperatures observed in Figures $5 \mathrm{c}$ and $\mathrm{d}$ were 231 and $138^{\circ} \mathrm{C}$. The corresponding movie file is provided in Supplementary Movie S2.

\section{CONCLUSION}

The novel, flexible, transparent film heaters developed herein exhibited a high heating performance up to $328^{\circ} \mathrm{C}$ using a $\mathrm{CFH}$ with $90 \%$ transparency and $0.058 \Omega \mathrm{sq}^{-1}$ sheet resistance. In addition, remarkable mechanical stretchability reaching $300 \%$ and stability after 1000 cycles of bending to the radius of $R_{\mathrm{b}}=1.3 \mathrm{~mm}$ were demonstrated without interruption of the Joule heating. The novel, costeffective approach based on electrospinning and electroplating enabled the formation of practically seamless junctions of copper-plated nanofibers at the intersection of fibers, which drastically reduced the junction resistance. Moreover, the overall nonwoven structure of the electrospun fibers formed a percolative network with significant interfiber distances, which greatly enhanced the transmittance and electrical conductivity of the film heaters. A rapid increase in surface temperature was demonstrated by the transparent heaters developed in this work using a minimal applied voltage of $1.5 \mathrm{~V}$, which could be supplied by portable batteries. The developed heating films are flexible and stretchable, and can be patterned and shaped as three-dimensional structures without losing their heating performance.

\section{ACKNOWLEDGEMENTS}

This research was supported by Global Frontier Program through the Global Frontier Hybrid Interface Materials (GFHIM) of the National Research Foundation of Korea (NRF) funded by the Ministry of Science, ICT \& Future Planning (2013M3A6B1078879) and the Technology Development Program to Solve Climate Changes of the National Research Foundation (NRF-2016M1A2A2936760). S.S. Yoon expresses his appreciation to the Vice Deanship of Scientific Research Chairs at King Saud University.

1 Fan, J. C. \& Bachner, F. J. Transparent heat mirrors for solar-energy applications. Appl. Opt. 15, 1012-1017 (1976).

2 Nishio, K., Sei, T. \& Tsuchiya, T. Preparation and electrical properties of ITO thin films by dip-coating process. J. Mater. Sci. 31, 1761-1766 (1996)

3 Alam, M. \& Cameron, D. Optical and electrical properties of transparent conductive ITO thin films deposited by sol-gel process. Thin Solid Films 377, 455-459 (2000). 
4 Mattox, D. M. Sol-gel derived, air-baked indium and tin oxide films. Thin Solid Films 204, 25-32 (1991).

5 Hamberg, I. \& Granqvist, C. G. Evaporated Sn-doped In203 films: basic optical properties and applications to energy-efficient windows. J. Appl. Phys. 60, R123-R160 (1986).

6 Kim, S.-S., Choi, S.-Y., Park, C.-G. \& Jin, H.-W. Transparent conductive ITO thin films through the sol-gel process using metal salts. Thin Solid Films 347, 155-160 (1999).

7 Leftheriotis, G., Papaefthimiou, S. \& Yianoulis, P. Development of multilayer transparent conductive coatings. Solid State lonics 136, 655-661 (2000).

8 Smyth, M., Eames, P. \& Norton, B. Annual performance of heat retaining integrated collector/storage solar water heaters in a northern maritime climate. Sol. Energy 70, 391-401 (2001).

9 Du Pasquier, A., Unalan, H. E., Kanwal, A., Miller, S. \& Chhowalla, M. Conducting and transparent single-wall carbon nanotube electrodes for polymer-fullerene solar cells. Appl. Phys. Lett. 87, 203511 (2005).

10 Williams, C. D., Robles, R. O., Zhang M., Li, S., Baughman, R. H. \& Zakhidov, A. A. Multiwalled carbon nanotube sheets as transparent electrodes in high brightness organic light-emitting diodes. Appl. Phys. Lett. 93, 183506 (2008).

11 Aguirre, C., Ternon, C., Paillet, M., Desjardins, P. \& Martel, R. Carbon nanotubes as injection electrodes for organic thin film transistors. Nano Lett. 9, 1457-1461 (2009).

$12 \mathrm{Im}$, K., Cho, K., Kim, J. \& Kim, S. Transparent heaters based on solution-processed indium tin oxide nanoparticles. Thin Solid Films 518, 3960-3963 (2010).

13 Bae, S., Kim, H., Lee, Y., Xu, X., Park, J.-S., Zheng, Y., Balakrishnan, J., Lei, T., Kim, H. R. \& Song, Y. I. Roll-to-roll production of 30-inch graphene films for transparent electrodes. Nat. Nanotechnol. 5, 574-578 (2010).

14 Kim, K. S., Zhao, Y., Jang, H., Lee, S. Y., Kim, J. M., Kim, K. S., Ahn, J.-H., Kim, P., Choi, J.-Y. \& Hong, B. H. Large-scale pattern growth of graphene films for stretchable transparent electrodes. Nat. 457, 706-710 (2009).

15 Zhang, M., Fang, S., Zakhidov, A. A., Lee, S. B., Aliev, A. E., Williams, C. D., Atkinson, K. R. \& Baughman, R. H. Strong, transparent, multifunctional, carbon nanotube sheets. Sci. 309, 1215-1219 (2005).

16 Hecht, D. S., Hu, L. \& Irvin, G. Emerging transparent electrodes based on thin films of carbon nanotubes, graphene, and metallic nanostructures. Adv. Mater. 23 1482-1513 (2011).

17 Vosgueritchian, M., Lipomi, D. J. \& Bao, Z. Highly conductive and transparent PEDOT: PSS films with a fluorosurfactant for stretchable and flexible transparent electrodes. Adv. Funct. Mater. 22, 421-428 (2012).

18 Gupta, R., Rao, K., Kiruthika, S. \& Kulkarni, G. U. Visibly transparent heaters. ACS Appl. Mater. Interfaces 8, 12559-12575 (2016).

19 Bae, J. J., Lim, S. C., Han, G. H., Jo, Y. W., Doung, D. L., Kim, E. S., Chae, S. J., Huy, T. Q., Van Luan, N. \& Lee, Y. H. Heat dissipation of transparent graphene defoggers. Adv. Funct. Mater. 22, 4819-4826 (2012).

20 Sui, D., Huang, Y., Huang, L., Liang, J., Ma, Y. \& Chen, Y. Flexible and transparent electrothermal film heaters based on graphene materials. Small 7, 3186-3192 (2011).

21 Kang, J., Kim, H., Kim, K. S., Lee, S.-K., Bae, S., Ahn, J.-H., Kim, Y.-J., Choi, J.-B. \& Hong, B. H. High-performance graphene-based transparent flexible heaters. Nano Lett. 11, 5154-5158 (2011).

22 Huang, Q., Shen, W., Fang, X., Chen, G., Guo, J., Xu, W., Tan, R. \& Song, W. Highly flexible and transparent film heaters based on polyimide films embedded with silver nanowires. RSC Adv. 5, 45836-45842 (2015).

23 Sorel, S., Bellet, D. \& Coleman, J. N. Relationship between material properties and transparent heater performance for both bulk-like and percolative nanostructured networks. ACS Nano 8, 4805-4814 (2014).

24 Hsu, P.-C., Liu, X., Liu, C., Xie, X., Lee, H. R., Welch, A. J., Zhao, T. \& Cui, Y. Personal thermal management by metallic nanowire-coated textile. Nano Lett. 15 365-371 (2014).

25 Gupta, R., Rao, K., Srivastava, K., Kumar, A., Kiruthika, S. \& Kulkarni, G. U. Spray coating of crack templates for the fabrication of transparent conductors and heaters on flat and curved surfaces. ACS Appl. Mater. Interfaces 6, 13688-13696 (2014).
26 Kim, T., Kim, Y. W., Lee, H. S., Kim, H., Yang, W. S. \& Suh, K. S. Uniformly interconnected silver-nanowire networks for transparent film heaters. Adv. Funct. Mater 23, 1250-1255 (2013).

27 Hong, S., Lee, H., Lee, J., Kwon, J., Han, S., Suh, Y. D., Cho, H., Shin, J., Yeo, J. \& Ko, S. H. Highly stretchable and transparent metal nanowire heater for wearable electronics applications. Adv. Mater. 27, 4744-4751 (2015).

28 Zhang, X., Yan, X., Chen, J. \& Zhao, J. Large-size graphene microsheets as a protective layer for transparent conductive silver nanowire film heaters. Carbon 69, 437-443 (2014).

29 Rao, K. \& Kulkarni, G. U. A highly crystalline single Au wire network as a high temperature transparent heater. Nanoscale 6, 5645-5651 (2014).

30 An, S., Jo, H. S., Kim, D.-Y., Lee, H. J., Ju, B.-K., Al-Deyab, S. S., Ahn, J.-H., Qin, Y. Swihart, M. T., Yarin, A. L. \& Yoon, S. S. Self-junctioned copper nanofiber transparent flexible conducting film via electrospinnig and electroplating. Adv. Mater. 28, 7149-7154 (2016).

31 Yarin, A. L., Pourdeyhimi, B. \& Ramakrishna, S. Fundamentals and Applications of Micro-and Nanofibers. (Cambridge University Press, Cambridge, UK, 2014).

32 Sinha-Ray, S., Zhang, Y. \& Yarin, A. L. Thorny devil nanotextured fibers: the way to cooling rates on the order of $1 \mathrm{~kW} / \mathrm{cm} 2$. Langmuir 27, 215-226 (2010).

33 Resnick, R., Halliday, D. \& Walker, J. Fundamentals of Physics vol. 1 (John Wiley, New York, NY, USA, 1988).

34 Jang, H.-S., Jeon, S. K. \& Nahm, S. H. The manufacture of a transparent film heater by spinning multi-walled carbon nanotubes. Carbon 49, 111-116 (2011).

35 Jung, D., Kim, D., Lee, K. H., Overzet, L. J. \& Lee, G. S. Transparent film heaters using multi-walled carbon nanotube sheets. Sens. Actuators, A 199, 176-180 (2013).

36 Kim, D., Lee, H.-C., Woo, J. Y. \& Han, C.-S. Thermal behavior of transparent film heaters made of single-walled carbon nanotubes. J. Phys. Chem. C 114 , 5817-5821 (2010).

37 Yoon, Y. H., Song, J. W., Kim, D., Kim, J., Park, J. K., Oh, S. K. \& Han, C. S Transparent film heater using single-walled carbon nanotubes. Adv. Mater. 19, 4284-4287 (2007).

38 Kwon, N., Kim, K., Heo, J., Yi, I. \& Chung, I. Study on Ag mesh/conductive oxide hybrid transparent electrode for film heaters. Nanotechnology 25, 265702 (2014).

39 Seong, B., Yoo, H., Nguyen, V. D., Jang, Y., Ryu, C. \& Byun, D. Metal-mesh based transparent electrode on a 3-D curved surface by electrohydrodynamic jet printing. J. Micromech. Microeng 24, 097002 (2014).

40 Kim, H.-J., Kim, Y., Jeong, J.-H., Choi, J.-H., Lee, J. \& Choi, D.-G. A cupronickel-based micromesh film for use as a high-performance and low-voltage transparent heater. J. Mater. Chem. A 3, 16621-16626 (2015).

41 Khan, A., Lee, S., Jang, T., Xiong, Z., Zhang, C., Tang, J., Guo, L. J. \& Li, W. D. Highperformance flexible transparent electrode with an embedded metal mesh fabricated by cost-effective solution process. Small 12, 3021-3030 (2016).

Attribution 4.0 International License. The images or other third party material in this article are included in the article's Creative Commons license, unless indicated otherwise in the credit line; if the material is not included under the Creative Commons license, users will need to obtain permission from the license holder to reproduce the material. To view a copy of this license, visit http:// creativecommons.org/licenses/by/4.0/

(C) The Author(s) 2017

Supplementary Information accompanies the paper on the NPG Asia Materials website (http://www.nature.com/am) 